Supporting information for:

\title{
Spectroscopic Characterization of Pt(IV) Terpyridyl Complexes
}

Stephen D. Taylor*, Vikas M. Shingade, Ronnie Muvirimi, Scott D. Hicks, Jeanette A. Krause, William B. Connick ${ }^{\#}$

Department of Chemistry, University of Cincinnati, P.O. Box 210172, Cincinnati, OH 452210172

\#deceased

*E-mail: Stephen@untoldcontent.com

\section{Contents}

Table S1. Summary of Crystal Data and Structure Refinement of the Pt(IV) Complexes. 2 Table S2: Select bond distances ( $\AA$ ) for the Pt(IV) complexes, Pt(II) analogs, and several octahedral $\mathrm{M}($ tpy $) \mathrm{X}_{3}(\mathrm{M}=\mathrm{Rh}, \mathrm{Ir} ; \mathrm{X}=\mathrm{Cl}, \mathrm{Br})$ complexes.

Table S3: UV-visible Absorption Data for [Pt(tpy)Cl](PF $)$, [Pt(tpy)Br]( $\left.\mathrm{PF}_{6}\right)$,

[Pt(tpy) $\left.\mathrm{Cl}_{3}\right]\left(\mathrm{PF}_{6}\right),\left[\mathrm{Pt}(\mathrm{tpy}) \mathrm{Br}_{3}\right]\left(\mathrm{PF}_{6}\right),\left[\mathrm{Pt}(\mathrm{tpy}) \mathrm{Br}_{3}\right]\left(\mathrm{Br}_{3}\right)$, tpy, and bromine in acetonitrile at room temperature.

Figure S1: ${ }^{1} \mathrm{H}$ NMR spectra of $[\mathrm{Pt}(\mathrm{tpy}) \mathrm{Br}]\left(\mathrm{PF}_{6}\right)+\mathrm{Br}_{2}(\mathrm{~A}),[\mathrm{Pt}(\mathrm{tpy}) \mathrm{Br}]\left(\mathrm{PF}_{6}\right)(\mathrm{B})$,

$[\mathrm{Pt}(\mathrm{tpy}) \mathrm{Cl}]\left(\mathrm{PF}_{6}\right)+\mathrm{Cl}_{2}(\mathrm{C})$, and $[\mathrm{Pt}(\mathrm{tpy}) \mathrm{Cl}]\left(\mathrm{PF}_{6}\right)(\mathrm{D})$ in DMF-d7.

Figure S2: Two-dimensional ${ }^{1} \mathrm{H}$ COSY NMR spectrum of $\left[\mathrm{Pt}(\mathrm{tpy}) \mathrm{Cl}_{3}\right]\left(\mathrm{PF}_{6}\right)$ in DMF-d7. 6 Figure S3: Two-dimensional ${ }^{1} \mathrm{H}$ COSY NMR spectrum of $\left[\mathrm{Pt}(\mathrm{tpy}) \mathrm{Br}_{3}\right]\left(\mathrm{PF}_{6}\right)$ in DMF-d7. 7 Figure S4: Crystal packing for [ $\left.\mathrm{Pt}(\mathrm{tpy}) \mathrm{Br}_{3}\right]\left(\mathrm{Br}_{3}\right)$.

Figure S5: $77 \mathrm{~K}$ emission spectra recorded of a $205 \mu \mathrm{M}$ solution of $\left[\mathrm{Pt}(\mathrm{tpy}) \mathrm{Cl}_{3}\right]\left(\mathrm{PF}_{6}\right)$ in butyronitrile at nine excitation wavelengths from 357 to $365 \mathrm{~nm}$.

Figure S6: Excitation spectra of $\left[\mathrm{Pt}\left(\mathrm{tpy}_{\mathrm{p}}\right) \mathrm{Br}_{3}\right]\left(\mathrm{PF}_{6}\right)\left(\lambda_{\mathrm{em}}=475 \mathrm{~nm}, 200 \mu \mathrm{M}\right)$ and $[\mathrm{Pt}(\mathrm{tpy}) \mathrm{Br}]\left(\mathrm{PF}_{6}\right)\left(\lambda_{\mathrm{em}}=475 \mathrm{~nm}, 117 \mu \mathrm{M}\right)$. 
Table S2. Summary of Crystal Data and Structure Refinement of the Pt(IV) Complexes.

\begin{tabular}{|c|c|c|c|c|}
\hline & {$\left[\mathrm{Pt}(\mathrm{tpy}) \mathrm{Br}_{3}\right]\left(\mathrm{PF}_{6}\right)$} & {$\left[\mathrm{Pt}(\mathrm{tpy}) \mathrm{Br}_{3}\right]\left(\mathrm{Br}_{3}\right)$} & {$\left[\mathrm{Pt}(\mathrm{tpy}) \mathrm{Br}_{3}\right] \mathrm{Br}$} & {$\left[\mathrm{Pt}(\mathrm{tpy}) \mathrm{Cl}_{3}\right]\left(\mathrm{PF}_{6}\right)$} \\
\hline CCDC Deposit No. & 1891068 & 1891069 & 1891070 & 1891071 \\
\hline Formula & {$\left[\mathrm{C}_{15} \mathrm{H}_{11} \mathrm{~N}_{3} \mathrm{Br}_{3} \mathrm{Pt}\right]\left(\mathrm{PF}_{6}\right)$} & {$\left[\mathrm{C}_{15} \mathrm{H}_{11} \mathrm{~N}_{3} \mathrm{Br}_{3} \mathrm{Pt}\right]\left(\mathrm{Br}_{3}\right)$} & {$\left[\mathrm{C}_{15} \mathrm{H}_{11} \mathrm{~N}_{3} \mathrm{Br}_{3} \mathrm{Pt}\right] \mathrm{Br}$} & {$\left[\mathrm{C}_{15} \mathrm{H}_{11} \mathrm{~N}_{3} \mathrm{Cl}_{3} \mathrm{Pt}\right]\left(\mathrm{PF}_{6}\right)$} \\
\hline Formula Weight & 813.06 & 907.82 & 748.00 & 679.68 \\
\hline Wavelength, $\AA$ & 0.77490 & 0.77490 & 0.77490 & 0.71073 \\
\hline Crystal System & Monoclinic & Hexagonal & Monoclinic & Monoclinic \\
\hline Space Group & $\mathrm{C} 2 / \mathrm{c}$ & $\mathrm{P} 6_{5}$ & $\mathrm{P} 2{ }_{1} / \mathrm{n}$ & $\mathrm{C} 2 / \mathrm{c}$ \\
\hline$a, \AA$ & $6.9107(8)$ & $8.7294(2)$ & $8.8447(11)$ & $6.6511(2)$ \\
\hline$b, \AA$ & $16.6503(19)$ & $8.7294(2)$ & $9.4371(12)$ & $16.6125(6)$ \\
\hline$c, \AA$ & $17.909(2)$ & $47.9200(16)$ & $21.434(3)$ & $17.7676(6)$ \\
\hline$a,^{\circ}$ & 90 & 90 & 90 & 90 \\
\hline$\beta, \circ$ & $96.251(2)$ & 90 & $99.026(2)$ & $96.5625(8)$ \\
\hline$\gamma,{ }^{\circ}$ & 90 & 120 & 90 & 90 \\
\hline$V, \AA^{3}$ & $2048.5(4)$ & $3162.36(19)$ & $1766.9(4)$ & $1950.30(12)$ \\
\hline$Z$ & 4 & 6 & 4 & 4 \\
\hline reflns collcd & 10913 & 31090 & 20688 & 28353 \\
\hline ind $\mathrm{reflns} / R_{\mathrm{int}}$ & $2087 / 0.0653$ & $5188 / 0.0621$ & $4342 / 0.0700$ & $2460 / 0.0269$ \\
\hline GOF on $F^{2}$ & 1.045 & 1.165 & 1.046 & 1.078 \\
\hline $\mathrm{R}_{1} / \mathrm{wR}_{2}[\mathrm{I}>2 \sigma(\mathrm{I})]^{\mathrm{a}}$ & $0.0302 / 0.0797$ & $0.0204 / 0.0444$ & $0.0340 / 0.0933$ & $0.0125 / 0.0276$ \\
\hline $\mathrm{wR}_{2}(\text { all data })^{\mathrm{a}}$ & $0.0351 / 0.0828$ & $0.0320 / 0.0467$ & $0.0350 / 0.0941$ & $0.0133 / 0.0278$ \\
\hline
\end{tabular}


Table S2. Select bond distances $(\AA)$ for the $\mathrm{Pt}(\mathrm{IV})$ complexes, $\mathrm{Pt}(\mathrm{II})$ analogs, and several octahedral $\mathrm{M}(\mathrm{tpy}) \mathrm{X}_{3}(\mathrm{M}=\mathrm{Rh}, \mathrm{Ir} ; \mathrm{X}=\mathrm{Cl}, \mathrm{Br})$ complexes.

\begin{tabular}{|c|c|c|c|c|}
\hline Compound $^{\mathrm{a}}$ & M-N(peripheral) & M-N (central) & M-X (axial) & M-X (equatorial) \\
\hline$\left[\mathrm{Pt}(\mathrm{tpy}) \mathrm{Cl}_{3}\right]\left(\mathrm{PF}_{6}\right)^{b}$ & $2.0328(15)$ & $1.962(2)$ & $2.3153(4)$ & $2.3076(6)$ \\
\hline$\left[\mathrm{Pt}(\mathrm{tpy}) \mathrm{Br}_{3}\right]\left(\mathrm{PF}_{6}\right)^{b}$ & $2.041(4)$ & $1.971(6)$ & $2.4664(6)$ & $2.4451(8)$ \\
\hline$\left[\mathrm{Pt}(\mathrm{tpy}) \mathrm{Br}_{3}\right]\left(\mathrm{Br}_{3}\right)^{b}$ & $2.044(5) / 2.032(5)$ & $1.962(6)$ & $2.4663(7) / 2.4649(7)$ & $2.4494(7)$ \\
\hline$\left[\mathrm{Pt}(\mathrm{tpy}) \mathrm{Br}_{3}\right] \mathrm{Br}^{b}$ & $2.035(4) / 2.042(4)$ & $1.965(4)$ & $2.4798(6) / 2.4649(5)$ & $2.4190(7)$ \\
\hline$[\mathrm{Pt}(\mathrm{tpy}) \mathrm{Cl}]\left(\mathrm{PF}_{6}\right)^{c}$ & $2.016(4) / 2.023(4)$ & $1.949(4)$ & na & $2.3058(10)$ \\
\hline$[\mathrm{Pt}(\mathrm{tpy}) \mathrm{Br}]\left(\mathrm{PF}_{6}\right)^{d}$ & $2.026(4)$ & $1.955(4)$ & na & $2.4266(5)$ \\
\hline$[\mathrm{Pt}(\mathrm{tpy}) \mathrm{Cl}] \mathrm{Cl}^{e}$ & $2.019(3) / 2.031(3)$ & $1.940(3)$ & na & $2.3044(10)$ \\
\hline$[\mathrm{Pt}(\mathrm{tpy}) \mathrm{Br}] \mathrm{Br}^{f}$ & $2.023(4) / 2.030(4)$ & $1.936(4)$ & na & $2.4270(5)$ \\
\hline $\mathrm{Rh}(\mathrm{tpy}) \mathrm{Cl}_{3} \mathrm{~g}$ & $2.042(4) / 2.052(4)$ & $1.959(4)$ & $2.3449(14) / 2.3451(14)$ & $2.3564(14)$ \\
\hline $\operatorname{Ir}(\operatorname{tpy}) \mathrm{Cl}_{3}{ }^{\mathrm{h}}$ & $2.044(3) / 2.049(3)$ & $1.927(3)$ & $2.3467(8) / 2.3556(8)$ & $2.3696(9)$ \\
\hline $\operatorname{Ir}(\operatorname{tpy}) \mathrm{Br}_{3}{ }^{\mathrm{h}}$ & $2.073(12) / 2.076(13)$ & $1.941(12)$ & $2.478(2) / 2.496(2)$ & $2.506(2)$ \\
\hline
\end{tabular}

${ }^{a}$ Data collected at $150 \mathrm{~K}$ for all Pt(IV) and Pt(II) complexes; ${ }^{\mathrm{b}}$ This work; ${ }^{\mathrm{c}}$ Taylor, S. D.; Howard, W.; Kaval, N.; Hart, R.; Krause, J. A.; Connick, W. B. Chem. Commun. 2010, 46, 1070; 'Krause, J.A.; Taylor, S.D.; Gerken, C.; Connick, W.B. CSD Communication, 2019, CCDC-1889549, CSD-Entry: SOBSOU, DOI: 10.5517/ccdc.csd.cc21f76y; ${ }^{\mathrm{e}}$ Krause, J.A.; Taylor, S.D.; Connick, W.B. CSD Communication, 2019, CCDC-1890126, CSD-Entry: FIVDIA, DOI:

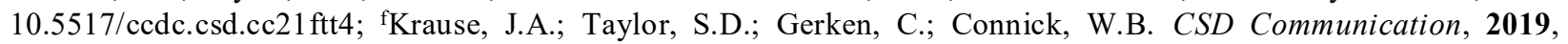
CCDC-1890127, CSD-Entry: FIVDOG, DOI: 10.5517/ccdc.csd.cc21ftv5; gPruchnik, F. P.; Jakimowicz, P.; Ciunik, Z.; Zakrzewska-Czerwinska, J.; Opolski, A.; Wietrzyk, J.; Wojdat, E. Inorg. Chim. Acta 2002, 334, 59; h Dobroschke, M.; Geldmacher, Y.; Ott, I.; Harlos, M.; Kater, L.; Wagner, L.; Gust, R.; Sheldrick, W. S.; Prokop, A. ChemMedChem 2009, 4, 177. 
Table S1. UV-visible Absorption Data for [Pt(tpy)Cl](PF6), $[\mathrm{Pt}(\mathrm{tpy}) \mathrm{Br}]\left(\mathrm{PF}_{6}\right),\left[\mathrm{Pt}\left(\mathrm{tpy}_{)} \mathrm{Cl}_{3}\right]\left(\mathrm{PF}_{6}\right)\right.$, $\left[\mathrm{Pt}\left(\mathrm{tpy}_{)}\right) \mathrm{Br}_{3}\right]\left(\mathrm{PF}_{6}\right),\left[\mathrm{Pt}(\mathrm{tpy}) \mathrm{Br}_{3}\right]\left(\mathrm{Br}_{3}\right)$, tpy, and bromine in acetonitrile at room temperature.

\begin{tabular}{|c|c|}
\hline Compound & $\operatorname{abs} \lambda_{\max } \mathrm{nm}\left(\varepsilon, \mathrm{M}^{-1} \mathrm{~cm}^{-1}\right)$ \\
\hline$[\mathrm{Pt}(\mathrm{tpy}) \mathrm{Cl}]\left(\mathrm{PF}_{6}\right)$ & $\begin{array}{l}207(30900), 254(30500), 270(22000), 281(30000), 305(10700), 318 \\
(11900), 331(17100), 348(9800), 378(2600), 392(2400)\end{array}$ \\
\hline$[\mathrm{Pt}(\mathrm{tpy}) \mathrm{Br}]\left(\mathrm{PF}_{6}\right)$ & $\begin{array}{l}197 \mathrm{sh}(37400), 231(23300), 256(24300), 273(19600), 283(26500), 306 \\
(10300), 320(11200), 334(15600), 349(7300), 379(2500), 400(2300)\end{array}$ \\
\hline$\left[\mathrm{Pt}(\mathrm{tpy}) \mathrm{Cl}_{3}\right]\left(\mathrm{PF}_{6}\right)$ & $\begin{array}{l}192 \text { (79 000), } 205 \text { (77 500), } 227 \text { (64 000), } 279 \text { sh (19 300), } 329 \text { sh (8 900), } \\
340 \text { (15 100), } 356 \text { (18 100) }\end{array}$ \\
\hline$\left[\mathrm{Pt}(\mathrm{tpy}) \mathrm{Br}_{3}\right]\left(\mathrm{PF}_{6}\right)$ & $\begin{array}{l}193(55600), 210(54300), 220 \mathrm{sh}(52400), 246(34600), 281 \text { sh }(21000), \\
289 \mathrm{sh}(15400), 329 \mathrm{sh}(9200), 340(12400), 357(13000), 397(800)\end{array}$ \\
\hline$\left[\mathrm{Pt}(\mathrm{tpy}) \mathrm{Br}_{3}\right]\left(\mathrm{Br}_{3}\right)$ & $\begin{array}{l}196(63700), 208(60000), 221(59200), 269 \text { (77 800), } 325 \text { (13 200), } 341 \\
(14900), 358(14300), 397(1700)\end{array}$ \\
\hline tpy & $\begin{array}{l}196(74400), 235(31500), 242 \text { sh }(28700), 278 \text { (30 100), } 302 \text { sh (20 500), } \\
313 \text { sh }(12500)\end{array}$ \\
\hline bromine & $196,240 \mathrm{sh}, 267,394$ \\
\hline
\end{tabular}




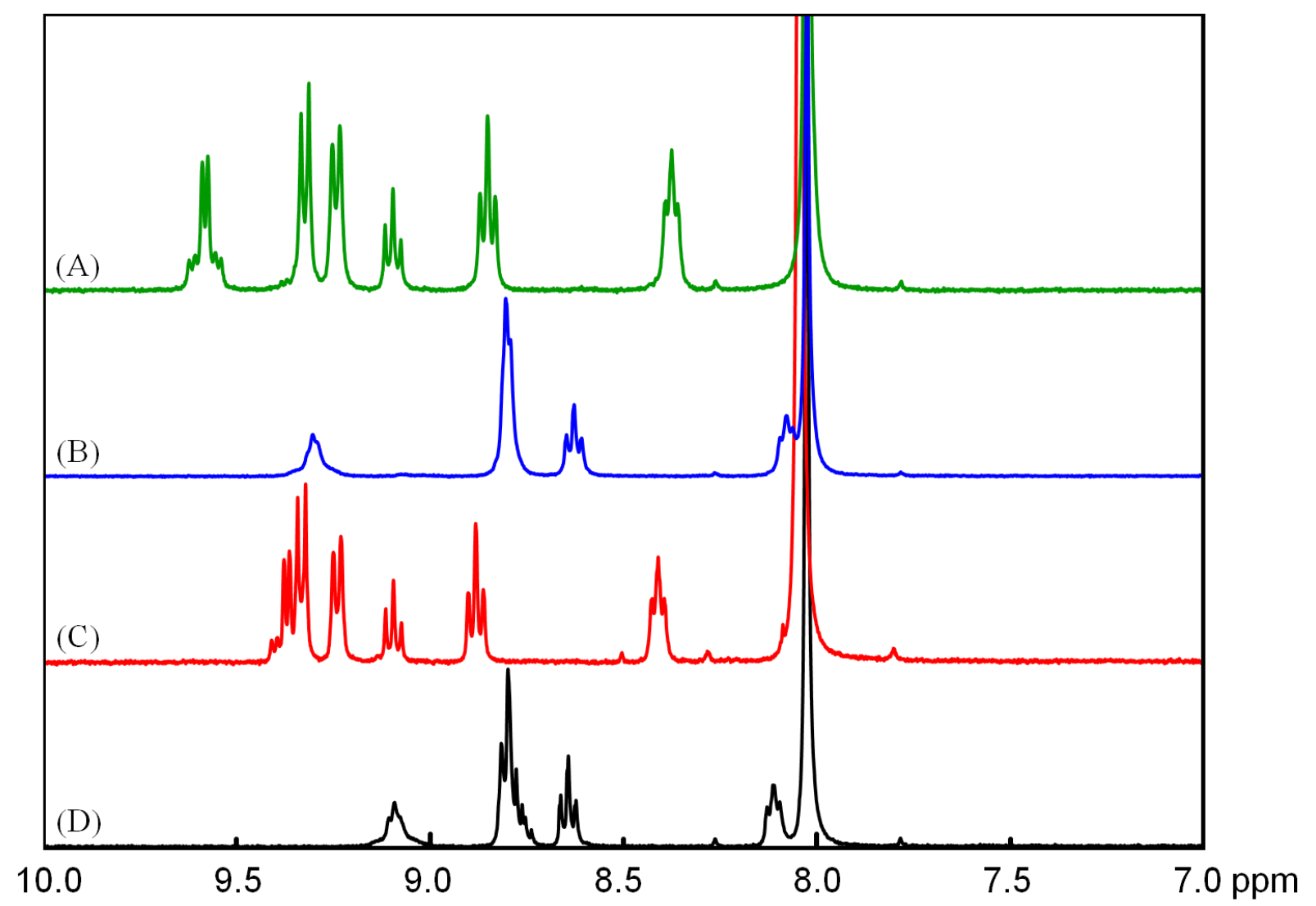

Figure S1. ${ }^{1} \mathrm{H}$ NMR spectra of $[\mathrm{Pt}(\mathrm{tpy}) \mathrm{Br}]\left(\mathrm{PF}_{6}\right)+\mathrm{Br}_{2}(\mathrm{~A},-$ - $)[\mathrm{Pt}(\operatorname{tpy}) \mathrm{Br}]\left(\mathrm{PF}_{6}\right)(\mathrm{B},-$ ), $[\mathrm{Pt}(\mathrm{tpy}) \mathrm{Cl}]\left(\mathrm{PF}_{6}\right)+\mathrm{Cl}_{2}(\mathrm{C},-)$, and $[\mathrm{Pt}(\mathrm{tpy}) \mathrm{Cl}]\left(\mathrm{PF}_{6}\right)(\mathrm{D},-)$ in $\mathrm{DMF}-\mathrm{d} 7$. 


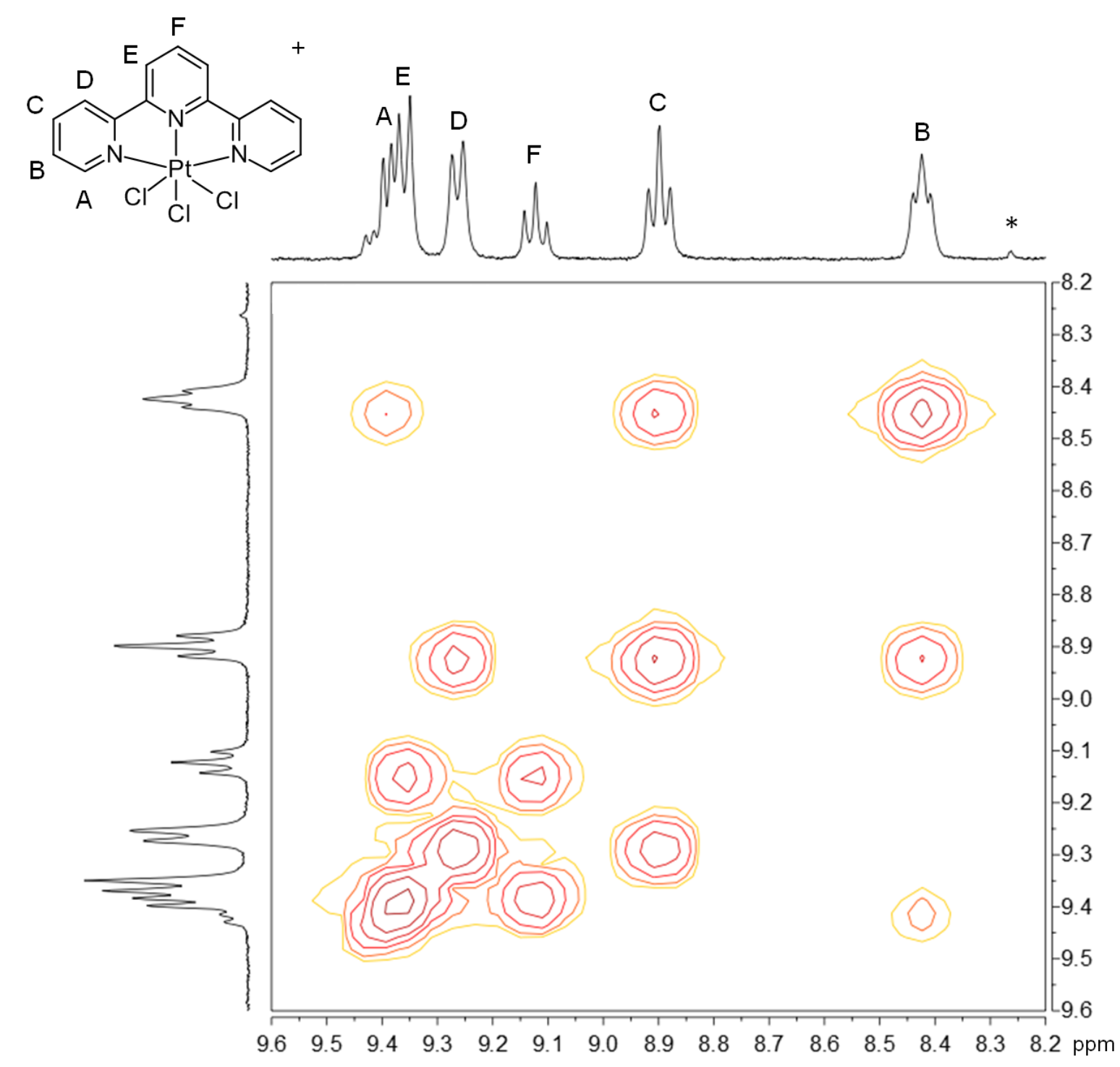

Figure S2. Two-dimensional ${ }^{1} \mathrm{H}$ COSY NMR spectrum of $\left[\mathrm{Pt}(\mathrm{tpy}) \mathrm{Cl}_{3}\right]\left(\mathrm{PF}_{6}\right)$ in DMF-d7. $\left({ }^{*}\right.$ marks resonances present in solvent blank.) 


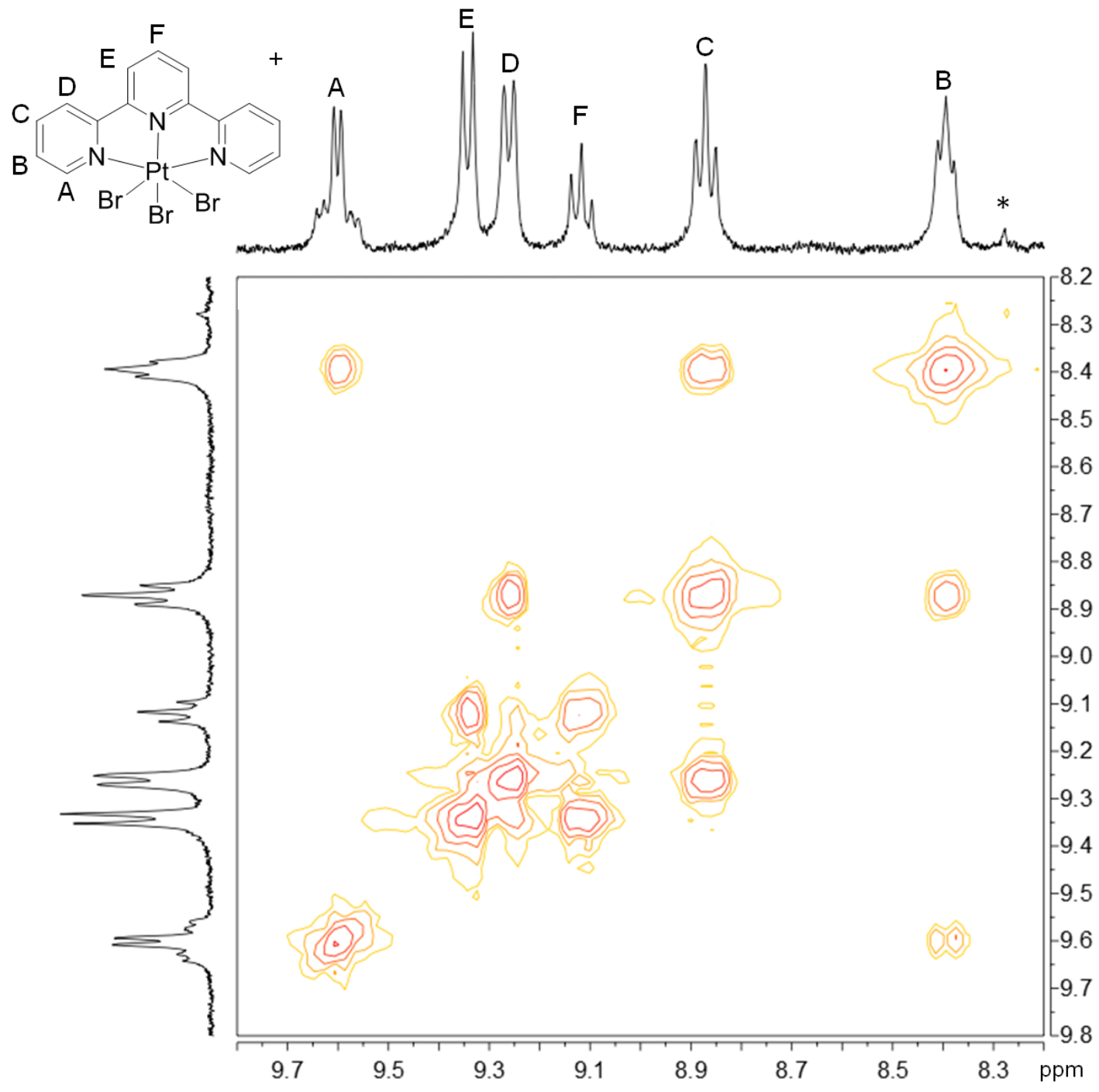

Figure S3. Two-dimensional ${ }^{1} \mathrm{H}$ COSY NMR spectrum of $\left[\mathrm{Pt}(\mathrm{tpy}) \mathrm{Br}_{3}\right]\left(\mathrm{PF}_{6}\right)$ in $\mathrm{DMF}-\mathrm{d}$ 7. (* marks resonances present in solvent blank.) 
(A)

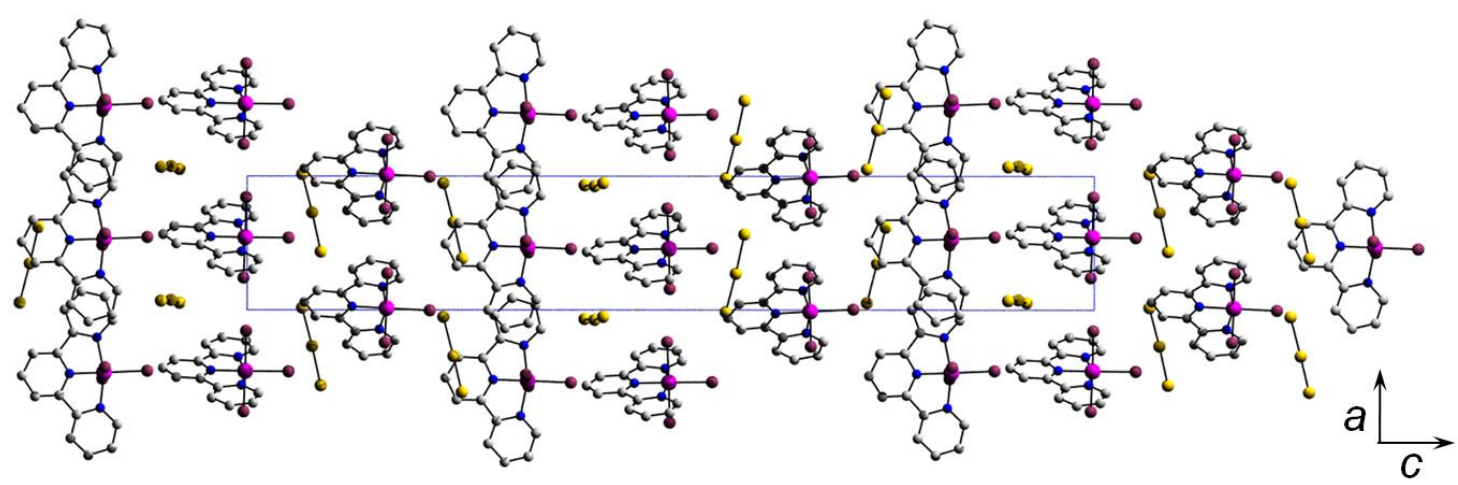

(B)

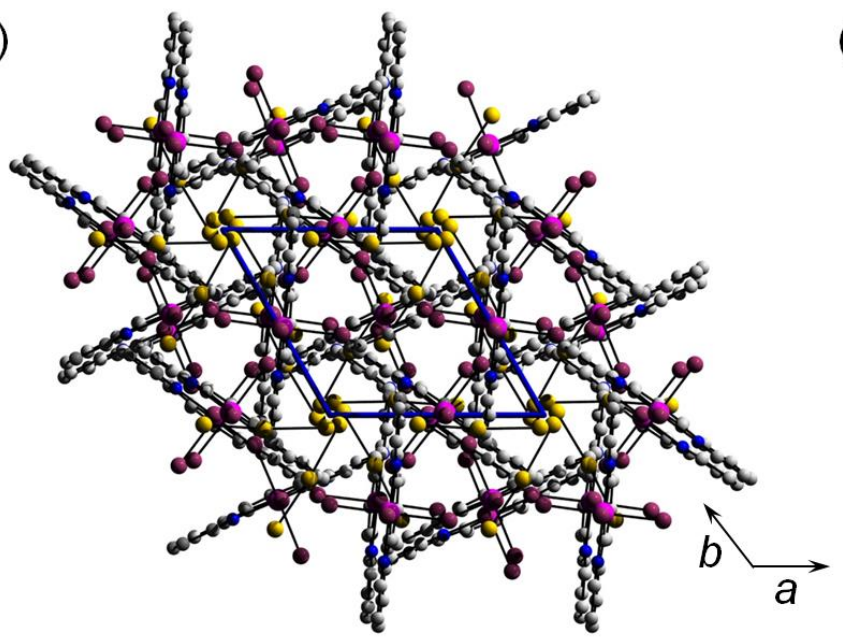

(C)

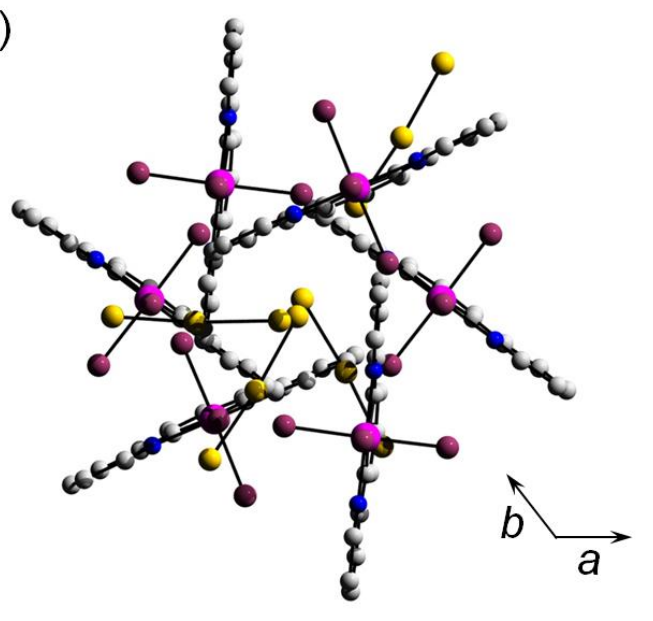

Figure S4. Crystal packing for [ $\mathrm{Pt}\left(\mathrm{tpyy}_{)} \mathrm{Br}_{3}\right]\left(\mathrm{Br}_{3}\right)$ 2.(Br3) (A) viewed perpendicular to the $a c$ plane, (B) packing arrangement viewed down the $c$ axis, and (C) hexagonal channels lined with tribromide anions viewed as one-half a unit cell length down the $c$ axis $\left(\mathrm{Br}^{-}\right.$in yellow), terminal $\operatorname{Br}(4)$ of the anion lies at the center of each channel. H-atoms omitted for clarity. 


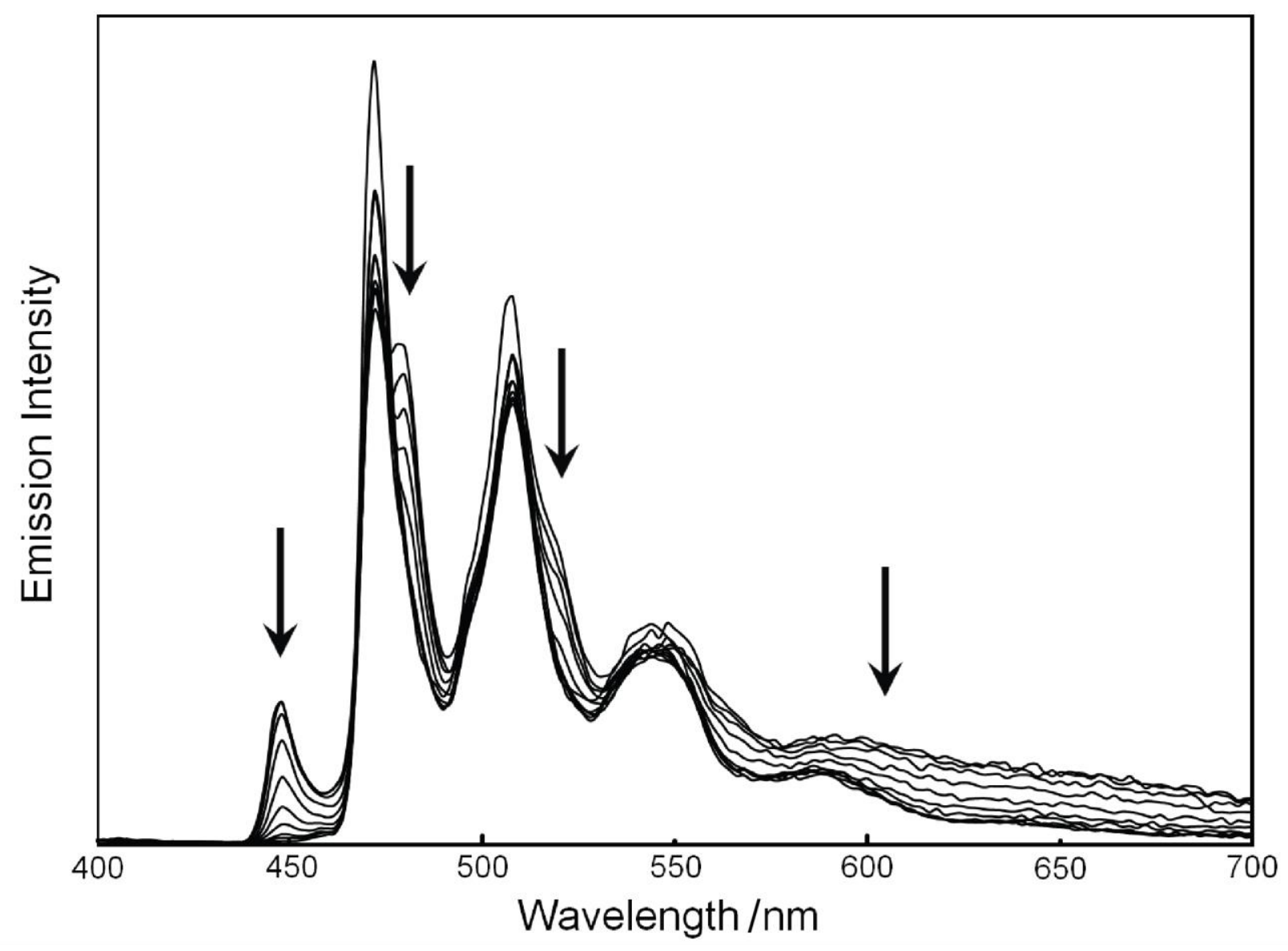

Figure S5. $77 \mathrm{~K}$ emission spectra recorded of a $205 \mu \mathrm{M}$ solution of $\left[\mathrm{Pt}(\mathrm{tpyy}) \mathrm{Cl}_{3}\right]\left(\mathrm{PF}_{6}\right)$ in butyronitrile at nine excitation wavelengths from 357 to $365 \mathrm{~nm}$ in $1 \mathrm{~nm}$ increments. Arrows indicate changes in the emission spectra with longer wavelength excitation. 


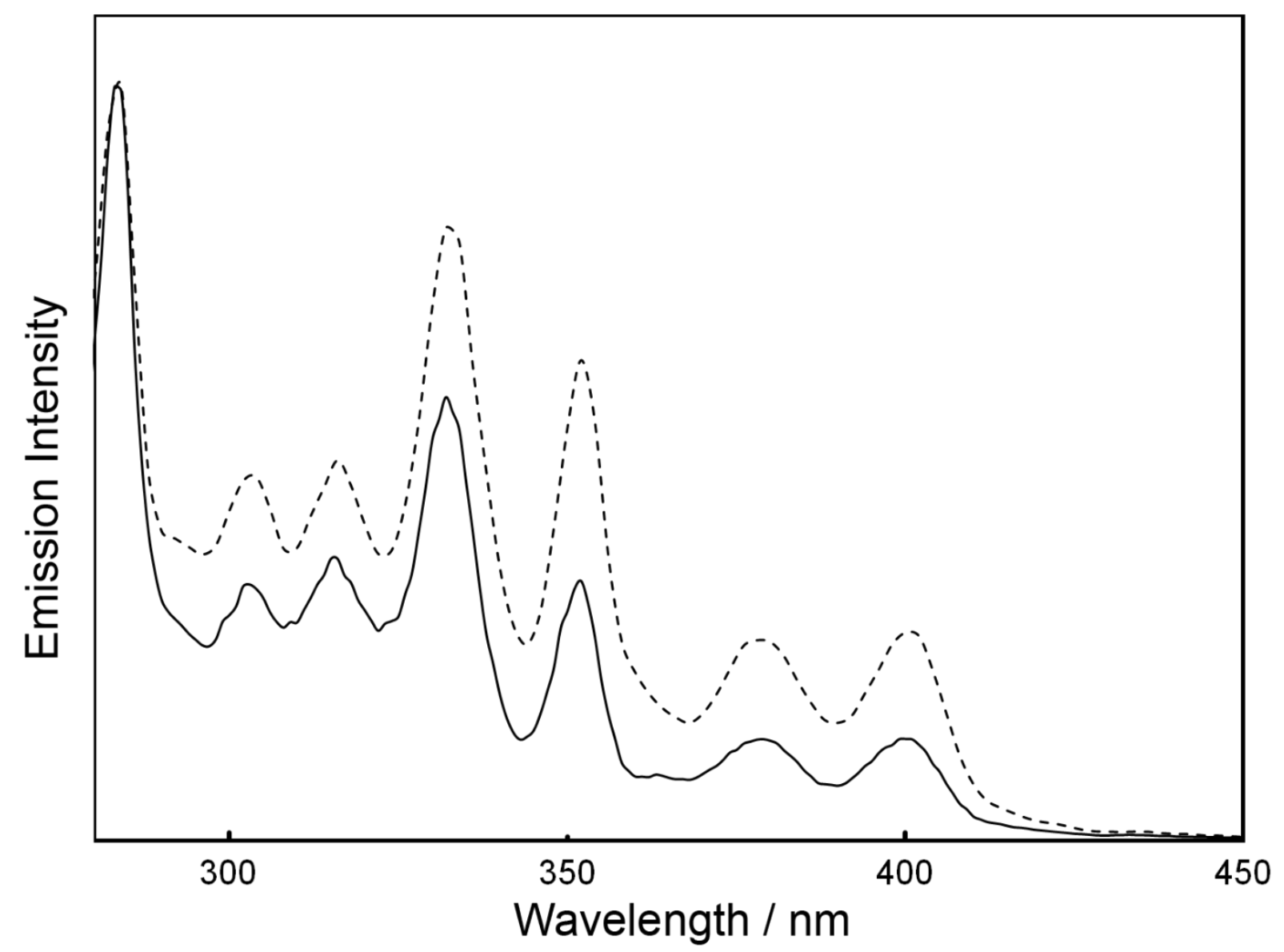

Figure S6. Excitation spectra of $\left[\mathrm{Pt}\left(\mathrm{tpy}_{\mathrm{p}}\right) \mathrm{Br}_{3}\right]\left(\mathrm{PF}_{6}\right)\left(-, \lambda_{\mathrm{em}}=475 \mathrm{~nm}, 200 \mu \mathrm{M}\right)$ and

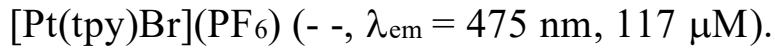

